\title{
Power System Contingency Analysis by using Voltage and Active Power Performance Index
}

\author{
Ummidi Sirisha ${ }^{1}$ M Sobha ${ }^{2}$ | Arun Kumar $\mathrm{Ch}^{3}$ \\ ${ }^{1}$ PG Scholar, Dept. of E.E.E., Sanketika Vidya Parishad Engineering College, Visakhapatnam, India. \\ ${ }^{2}$ Assistant Professor, Dept. of E.E.E., Sanketika Vidya Parishad Engineering College, Visakhapatnam, India. \\ ${ }^{3}$ Assistant Professor, Dept. of E.E.E., Dr L B Engineering College for Women, Visakhapatnam, India.
}

\section{To Cite this Article}

Ummidi Sirisha, M Sobha and Arun Kumar Ch, "Power System Contingency Analysis by using Voltage and Active Power Performance Index", International Journal for Modern Trends in Science and Technology, Vol. 06, Issue 07, July 2020, pp.:52-60; https://doi.org/10.46501/IJMTST060708

\section{Article Info}

Received on 11-June-2020, Revised on 18-June-2020, Accepted on 21-June-2020, Published on 30-June-2020.

\section{ABSTRACT}

Now a days, power system protection is an important task for an operating engineer, which can be done by doing online security assessment. The contingency analysis technique is a prerequisite to predict the effects of various contingencies like failure of transformers, transmission lines, etc. To do contingency analysis first the operator has to know the parameters like voltage, power and voltage angle at each and every bus by doing load flow analysis on the system. Newton Raphson method is the best load flow method as it gives accurate results in less time. In this paper for each line outage contingency, load flow analysis has been done on the system and the active power and voltage performance indices have been calculated. These two performance indices will give the idea about the change in active power flow through the lines and voltages at the buses for a particular line outage. Summation of these two indices will give the performance index value through which ranking of severity will be given to the lines. Based on this, the ranking of most severe contingency has been done based on the values of performance indices. Simultaneously the value of bus voltages and active power flow before and after the most severe transmission line contingency has been analyzed. The effectiveness of the method has been tested on 5-Bus, 6-Bus, IEEE-14 Bus and IEEE-30 Bus test systems in MATLAB environment. This contingency analysis helps the operational engineer to know which line outage is dangerous to the system and what prior action is to be taken to minimize the effect of that particular line outage.

KEYWORDS:- Contingency selection, Newton Raphson Method, Performance index, overload.

Copyright (C) 2014-2020 International Journal for Modern Trends in Science and Technology

DOI: https://doi.org/10.46501/IJMTST060708

\section{INTRODUCTION}

Contingency is expressed as an unwanted event occurring in the power system for a short duration of time, which actually specify the loss or outage of one or more components of power system [1]. At the time of outage of any component or equipment in the power system, contingency analysis shows an indication, of what might be the position of power system [2]. It is fundamentally a software application run in power management system, simulating a speculative test on a list of notional cases, which would create power flow, voltage or reactive power violations in the sytem. These cases are recognized and ranked in order of their severity using contingency ranking approach [3]. Generally contingency analysis is separated into three parts, 
contingency creation, selection, and evaluation [4], but now a days the selection and the evaluation both are doing in same section. Many work has been done on contingency selection mainly, whose aim is to reduce the indigenous long list of contingencies by selecting only the outages with severe limit violations [5]. This selection is seasoned by mainly two methods, i.e. contingency ranking and contingency screening. The screening methods are local solution based study, which primarily gives importance to the most dangerous cases for complete ac analysis, simultaneously the non-severe cases are deleted from the list [6]. Alternative method is ranking method, which uses performance index as a scalar value to narrate the effects of an outage on the whole system [7]. In this work, the effort has been given on contingency ranking. At starting the contingency list is formed, which contains those cases whose probability of getting outage is found to be high. The list, which is generally large, is translated into electrical network changes, mostly generator and/or line outages. Contingency evaluation using load flow is then carrying out on the following individual outages in decreasing order of severity. The activity will be remained up to the point where no post contingency violations are undergone.

\section{CONTINGENCY ANALYSIS}

\section{A. Contingency Creation}

It is the initial step of contingency analysis. It is made up of all set of viable contingencies that may happen in a power system. This process consists of making contingency lists.

\section{B. Contingency Selection}

It is the second step in contingency analysis; it is the process which includes finding of severe contingencies from all that may cause to violate bus voltages and power through lines. Here in this procedure contingency list is reduced by rejection of least severe contingency and taking into consideration of most severe outages. In this process the performance index has been used to find the most severe ones.

\section{Contingency Evaluation}

It is the third step and the most significant step as it includes necessary control and security actions which are required in order to reduce the effects of most severe contingencies in a power system.

\section{UTILIZED LOAD FLOW METHOD}

The Newton-Raphson method is the most useful method for load flow solutions because of its different advantages. It has dominant convergence characteristics compared to remaining load flow methods. And has less calculation. The NewtonRaphson method is benefit for large power system networks as because computer storage requirements are less and increases almost linearly with size of problem.

The following equations are used in $\mathrm{N}-\mathrm{R}$ method to measure voltage, current, active power and reactive power at different buses.

The current injected at the bus $\mathrm{m}$ is

$$
I_{m}=\sum_{l=1}^{n} Y_{m l} V_{l}
$$

Where $\mathrm{n}$ is the number of buses in the system.

$Y_{m l}$ is the admittance between lines $\mathrm{m}$ and 1 .

$V_{l}$ is the voltage at bus $\mathrm{I}$.

In polar form we can write equation 1 as

$$
I_{m}=\sum_{l=1}^{n}\left|Y_{m l}\right|\left|V_{l}\right| \angle\left(\theta_{m l}+\delta_{l}\right)
$$

Where $\theta_{m l}$ is the admittance angle between lines $\mathrm{m}$ and 1 .

$\delta_{l}$ is the voltage angle of bus 1.

We can write current in terms of active and reactive Power

$$
I_{m}=\frac{P_{m}-j Q_{m}}{V_{m}^{*}}
$$

Where $P_{m}$ is the active power injected into the system from bus $m$.

$Q_{m}$ is the reactive power injected into the system from bus $\mathrm{m}$.

$V_{m}$ is the voltage magnitude at bus $\mathrm{m}$.

After substitution of $I_{m}$ value in equation 3 we can get

$$
P_{m}-j Q_{m}=\left|V_{m}\right| \angle-\delta_{m} \sum_{l=1}^{n}\left|Y_{m l}\right|\left|V_{l}\right| \angle\left(\theta_{m l}+\delta_{l}\right)
$$

By separating real and imaginary terms, we get

$$
\begin{aligned}
& P_{m}=\sum_{l=1}^{n}\left|Y_{m l}\right|\left|V_{l}\right|\left|V_{m}\right| \operatorname{Cos}\left(\theta_{m l}-\delta_{m}+\delta_{l}\right) \\
& Q_{m}=\sum_{l=1}^{n}\left|Y_{m l}\right|\left|V_{l}\right|\left|V_{m}\right| \operatorname{Sin}\left(\theta_{m l}-\delta_{m}+\delta_{l}\right)
\end{aligned}
$$

By neglecting higher order terms if we expand equation 5 and 6 by using Taylor's series we will get 


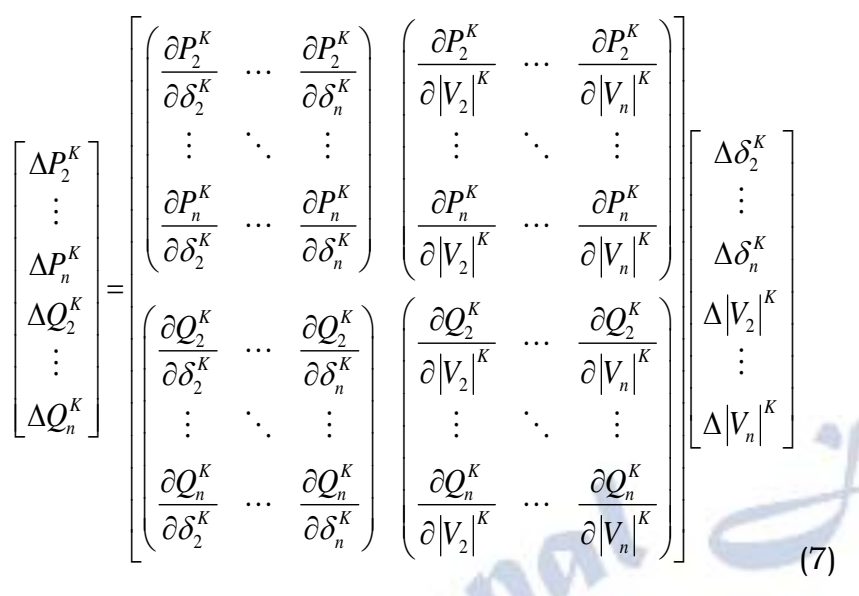

The jacobian matrix will give the linear relationship between small changes in the angle $\Delta \delta_{i}^{K}$ and change in bus voltage $\Delta V_{i}^{K}$ with small variations in real power and reactive power $\Delta P_{i}^{K}$ and $\Delta Q_{i}^{K}$

$$
\left(\begin{array}{l}
\Delta P \\
\Delta Q
\end{array}\right)=\left(\begin{array}{ll}
J_{1} & J_{2} \\
J_{3} & J_{4}
\end{array}\right)\left(\begin{array}{c}
\Delta \delta \\
\Delta|V|
\end{array}\right)
$$

The diagonal and off diagonal elements of $\mathrm{J}_{1}$ are

$$
\begin{aligned}
& \frac{\partial P_{m}}{\partial \delta_{m}}=\sum_{l \neq m}\left|V_{m}\right|\left|V_{l}\right|\left|Y_{l m}\right| \operatorname{Sin}\left(\theta_{m l}-\delta_{m}+\delta_{l}\right) \\
& \frac{\partial P_{m}}{\partial \delta_{m}}=-\left|V_{m}\right|\left|V_{l}\right|\left|Y_{m l}\right| \operatorname{Sin}\left(\theta_{m l}-\delta_{m}+\delta_{l}\right), l \neq m
\end{aligned}
$$

The power residuals $\Delta P_{m}^{K}, \Delta Q_{m}^{K}$ can be written as

$$
\begin{aligned}
& \Delta P_{m}^{K}=P_{m}^{s c h}-P_{m}^{K} \\
& \Delta Q_{m}^{K}=Q_{m}^{s c h}-Q_{m}^{K}
\end{aligned}
$$

Where $P_{m}^{s c h}$ is the scheduled active power at bus $\mathrm{m}$.

$Q_{m}^{s c h}$ is the scheduled reactive power at bus m.

From the power residuals and jacobian matrix we can get the voltage magnitude $\left|V_{m}^{K}\right|$ and voltage angle $\delta_{m}^{K}$.

The new values of voltages and angles are

$$
\begin{aligned}
& \left|V_{m}^{(K+1)}\right|=\left|V_{m}^{K}\right|+\Delta\left|V_{m}^{K}\right| \\
& (12) \\
& \delta_{m}^{(K+1)}=\delta_{m}^{K}+\Delta \delta_{m}^{K}
\end{aligned}
$$

\section{SYSTEM PERFORMANCE INDEX}

The deviation of power system variables like bus voltages, power flows, from its rated value is measured by the system performance index. It is also used to evaluate the relative stability of a contingency [8].

1. Voltage performance index $\left(\mathrm{PI}_{\mathrm{V}}\right)$

2. Active power performance index $\left(\mathrm{PI}_{\mathrm{Mw}}\right)$

A. Voltage Performance Index (PIV):

The power system deficiency due to violation of bus voltages is described by the voltage performance index [2].

$$
P I_{v}=\sum_{i=1}^{n}\left(\frac{W}{2 z}\right)\left\{\frac{\left(\left|V_{i}\right|-\left|V_{i}^{s p}\right|\right)}{\Delta V_{i}^{\lim }}\right\}^{2 z}
$$

Where $\left|V_{i}\right|$ is the ith bus voltage magnitude.

$\left|V_{i}^{s p}\right|$ is the $\mathrm{i}^{\text {th }}$ bus specified voltage magnitude.

$\Delta V_{i}^{\lim }$ is the voltage deviation limit which we can measure by taking average value of minimum and maximum allowable voltages at bus $i$.

$z$ is exponent of the penalty function and value is $(=1)$.

$\mathrm{n}$ is the number of buses in the given power system.

$\mathrm{W}$ is the real non negative weighting factor and value is $(=1)$

Here to calculate $\Delta V_{i}^{\mathrm{lim}}$ maximum voltage limit is 1.05 p.u and minimum voltage limit is 0.95 p.u since $\pm 5 \%$ deviation in voltage is allowed. This voltage performance index will give the information about the change in voltage at each and every bus.

B. Active Power Performance Index $\left(\mathrm{PI}_{\mathrm{Mw}}\right)$ :

For identify the power over flows through the transmission lines the indices used is active power performance index [9].

$$
P I_{M W}=\sum_{i=1}^{N_{L}}\left(\frac{W}{2 z}\right)\left\{\frac{P_{l}}{P_{l}^{\max }}\right\}^{2 z}
$$

Where, $P_{l}$ is the power flow (MW) through the line 1.

$P_{l}^{\max }$ is the maximum capacity of power flow through the line 1 .

$N_{L}$ is the number of the transmission lines of the power system 
$\mathrm{W}$ is the real non-negative weighting facto and value is $(=1)$

$z$ is exponent of penalty function and value is $(=1)$

$$
P_{l}^{\max }=\frac{V_{i}^{*} V_{j}}{X}
$$

Where $V_{i}$ is the voltage at bus $i$.

$V_{j}$ is the voltage at bus $\mathrm{j}$.

$X$ is the reactance of the line between bus $i$ and bus $j$.

\section{TEST CASES}

To perform contingency analysis, IEEE standard 5 bus, 6 bus, 14 bus and30 bus systems are considered.

\section{Test case 1: 5 BUS SYSTEM}

TABLE 1.1: SYSTEM DATA

\begin{tabular}{|c|c|c|c|c|c|}
\hline $\begin{array}{c}\text { From } \\
\text { Bus }\end{array}$ & $\begin{array}{c}\text { To } \\
\mathrm{Bu} \\
\mathrm{s}\end{array}$ & $\begin{array}{c}\mathrm{R} \\
\text { (p.u.) }\end{array}$ & $\begin{array}{c}\mathrm{X} \\
\text { (p.u.) }\end{array}$ & $\begin{array}{c}\mathrm{B} / 2 \\
\text { (p.u.) }\end{array}$ & $\begin{array}{c}\text { Real Power } \\
\text { Capacity(p.u.) }\end{array}$ \\
\hline 1 & 2 & 0.02 & 0.06 & 0.030 & 0.8 \\
\hline 1 & 3 & 0.08 & 0.24 & 0.025 & 0.3 \\
\hline 2 & 3 & 0.06 & 0.25 & 0.020 & 0.2 \\
\hline 2 & 4 & 0.06 & 0.18 & 0.020 & 0.2 \\
\hline 2 & 5 & 0.04 & 0.12 & 0.015 & 0.6 \\
\hline 3 & 4 & 0.01 & 0.03 & 0.010 & 0.1 \\
\hline 4 & 5 & 0.08 & 0.24 & 0.025 & 0.1 \\
\hline
\end{tabular}

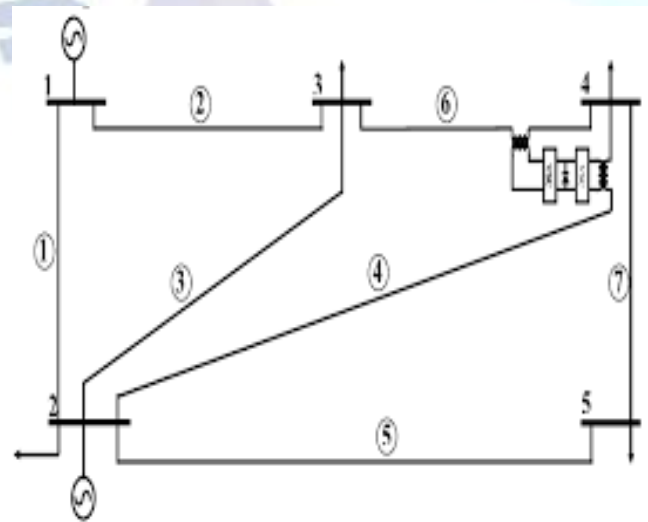

Fig. I: Five-bus Network

For analysis IEEE standard 5 bus system is considered. Bus 1 is slack bus, bus 2 is generator and 3,4 , and 5 are load buses. Now for each line outage the load flow method has been carried out and the active power performance index and voltage performance index have been calculated using equations 14 and 15 . The ranking has been given based on the Performance Index (P. I).
TABLE 1.2: RANKING OF THE LINES for 5-bus system

\begin{tabular}{|c|c|c|c|c|}
\hline $\begin{array}{c}\text { Tripped } \\
\text { line }\end{array}$ & $\mathrm{PI}_{\mathrm{V}}$ & $\mathrm{PI}_{\mathrm{MW}}$ & $\mathrm{PI}$ & Rank \\
\hline $1-2$ & 2.4587 & 0.1386 & 2.5973 & 3 \\
\hline $1-3$ & 3.2521 & 0.0276 & 3.2797 & 2 \\
\hline $2-3$ & 2.2540 & 0.0295 & 2.2834 & 6 \\
\hline $2-4$ & 2.3784 & 0.0293 & 2.4077 & 4 \\
\hline $2-5$ & 11.1376 & 0.0811 & 11.2186 & 1 \\
\hline $3-4$ & 2.2836 & 0.0235 & 2.3070 & 5 \\
\hline $4-5$ & 2.1436 & 0.0230 & 2.1666 & 7 \\
\hline
\end{tabular}

TABLE 1.3: PRE CONTINGENCY AND POST CONTINGENCY STATE BUS VOLTAGES FOR THE LINE OUTAGE (2-5)

\begin{tabular}{|c|c|c|}
\hline $\begin{array}{c}\text { Bus } \\
\text { No. }\end{array}$ & $\begin{array}{c}\text { Pre-Contingency } \\
\text { Voltage } \\
\text { (p.u.) }\end{array}$ & $\begin{array}{c}\text { Post-Contingency } \\
\text { Voltage } \\
\text { (p.u.) }\end{array}$ \\
\hline 1 & 1.0600 & 1.0600 \\
\hline 2 & 1.0000 & 1.0000 \\
\hline 3 & 0.9873 & 0.9602 \\
\hline 4 & 0.9841 & 0.9503 \\
\hline 5 & 0.9717 & 0.8579 \\
\hline
\end{tabular}

TABLE 1.4: POST CONTINGENCY POWERFLOWS

\begin{tabular}{|c|c|c|c|}
\hline \multirow{2}{*}{$\begin{array}{c}\text { From } \\
\text { Bus }\end{array}$} & \multirow{2}{*}{$\begin{array}{c}\text { To } \\
\text { Bus }\end{array}$} & $\begin{array}{l}\text { When } 2-5 \\
\text { line is } \\
\text { tripped }\end{array}$ & \multirow{2}{*}{$\begin{array}{c}\text { Real } \\
\text { Power } \\
\text { Capacity } \\
\text { (p.u.) }\end{array}$} \\
\hline & & $\begin{array}{c}\text { Post- } \\
\text { Contingency } \\
\text { Power (p.u.) }\end{array}$ & \\
\hline 1 & 2 & 0.8147 & 0.8 \\
\hline 1 & 3 & 0.5604 & 0.3 \\
\hline 2 & 3 & 0.4517 & 0.2 \\
\hline 2 & 4 & 0.5399 & 0.2 \\
\hline 2 & 5 & 0 & 0.6 \\
\hline 3 & 4 & 0.5215 & 0.1 \\
\hline 4 & 5 & 0.6398 & 0.1 \\
\hline
\end{tabular}

After the (2-5) line is tripped, all the transmission lines are overloaded as shown in the above table.

\section{Test case 2: 6 BUS SYSTEM}

TABLE 2.1: SYSTEM DATA

\begin{tabular}{|c|c|c|c|c|c|}
\hline $\begin{array}{c}\text { From } \\
\text { Bus }\end{array}$ & $\begin{array}{c}\text { To } \\
\text { Bus }\end{array}$ & $\begin{array}{c}\mathrm{R} \\
\text { (p.u.) }\end{array}$ & $\begin{array}{c}\mathrm{X} \\
\text { (p.u.) }\end{array}$ & $\begin{array}{c}\mathrm{B} / 2 \\
\text { (p.u.) }\end{array}$ & $\begin{array}{c}\text { Real Power } \\
\text { Capacity } \\
\text { (p.u.) }\end{array}$ \\
\hline 1 & 2 & 0.10 & 0.20 & 0.02 & 0.3 \\
\hline 1 & 4 & 0.05 & 0.20 & 0.02 & 0.5 \\
\hline 1 & 5 & 0.08 & 0.30 & 0.03 & 0.4 \\
\hline 2 & 3 & 0.05 & 0.25 & 0.03 & 0.2 \\
\hline 2 & 4 & 0.05 & 0.10 & 0.01 & 0.4 \\
\hline
\end{tabular}




\begin{tabular}{|c|c|c|c|c|c|}
\hline 2 & 5 & 0.10 & 0.30 & 0.02 & 0.2 \\
\hline 2 & 6 & 0.07 & 0.20 & 0.025 & 0.3 \\
\hline 3 & 5 & 0.12 & 0.30 & 0.025 & 0.2 \\
\hline 3 & 6 & 0.02 & 0.10 & 0.01 & 0.6 \\
\hline 4 & 5 & 0.20 & 0.40 & 0.04 & 0.2 \\
\hline 5 & 6 & 0.10 & 0.30 & 0.03 & 0.2 \\
\hline
\end{tabular}

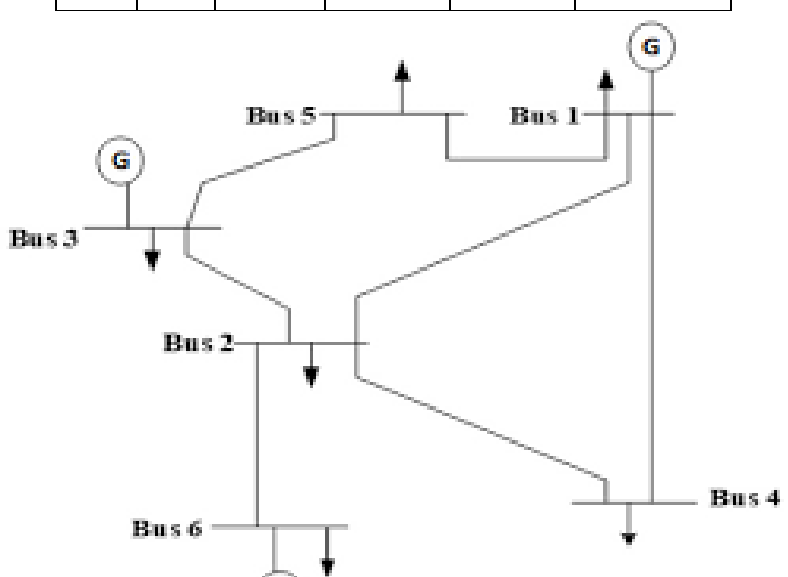

(G)

Fig.2: Six-bus Network

For analysis IEEE standard 6 bus system is considered. Bus 1 is slack bus, 2, 3 buses are generator and 4, 5, and 6 are load buses. Now for each line outage the load flow method has been carried out and the active power performance index and voltage performance index has been calculated using equations 14 and 15 . The ranking has been given based on the Performance Index ( P. I).

\section{TABLE 2.2: RANKING OF THE LINES}

\begin{tabular}{|c|c|c|c|c|}
\hline Tripped line & $\mathrm{PI}_{\mathrm{V}}$ & $\mathrm{PI}_{\mathrm{MW}}$ & $\mathrm{PI}$ & Rank \\
\hline $1-2$ & 4.0842 & 0.0455 & 4.1296 & 11 \\
\hline $1-4$ & 5.0979 & 0.0561 & 5.1539 & 3 \\
\hline $1-5$ & 4.7019 & 0.0511 & 4.7530 & 5 \\
\hline $2-3$ & 4.0974 & 0.0337 & 4.1311 & 10 \\
\hline $2-4$ & 8.9559 & 0.0500 & 9.0059 & 2 \\
\hline $2-5$ & 4.5972 & 0.0384 & 4.6356 & 6 \\
\hline $2-6$ & 4.2595 & 0.0396 & 4.2991 & 7 \\
\hline $3-5$ & 4.9139 & 0.0421 & 4.9560 & 4 \\
\hline $3-6$ & 9.6332 & 0.0811 & 9.7143 & 1 \\
\hline $4-5$ & 4.1636 & 0.0344 & 4.1980 & 9 \\
\hline $5-6$ & 4.2344 & 0.0335 & 4.2679 & 8 \\
\hline
\end{tabular}

TABLE 2.3: PRE CONTINGENCY AND POST CONTINGENCY STATE BUS VOLTAGES FOR THE LINE OUTAGE (3-6)

\begin{tabular}{|c|c|c|}
\hline Bus No. & $\begin{array}{c}\text { Pre-Contingency } \\
\text { Voltage } \\
\text { (p.u.) }\end{array}$ & $\begin{array}{c}\text { Post-Contingency } \\
\text { Voltage } \\
\text { (p.u.) }\end{array}$ \\
\hline 1 & 1.0500 & 1.0500 \\
\hline 2 & 1.0500 & 1.0500 \\
\hline 3 & 1.0700 & 1.0700 \\
\hline 4 & 0.9894 & 0.9854 \\
\hline 5 & 0.9854 & 0.9574 \\
\hline 6 & 1.0044 & 0.8898 \\
\hline
\end{tabular}

\section{TABLE 2.4: POST CONTINGENCY POWERFLOWS}

\begin{tabular}{|c|c|c|c|}
\hline \multirow{2}{*}{$\begin{array}{c}\text { From } \\
\text { Bus }\end{array}$} & \multirow[b]{2}{*}{ To Bus } & $\begin{array}{c}\text { When 3-6 } \\
\text { line is } \\
\text { tripped }\end{array}$ & \multirow{2}{*}{$\begin{array}{c}\text { Real } \\
\text { Power } \\
\text { Capacity } \\
\text { (p.u.) }\end{array}$} \\
\hline & & \begin{tabular}{|c|} 
Post- \\
Contingend \\
y Power \\
(p.u.)
\end{tabular} & \\
\hline 1 & 2 & 0.3218 & 0.3 \\
\hline 1 & 4 & 0.4602 & 0.5 \\
\hline 1 & 5 & 0.3626 & 0.4 \\
\hline 2 & 3 & 0.2132 & 0.2 \\
\hline 2 & 4 & 0.3187 & 0.4 \\
\hline 2 & 5 & 0.1500 & 0.2 \\
\hline 2 & 6 & 0.5550 & 0.3 \\
\hline 3 & 5 & 0.3847 & 0.2 \\
\hline 3 & 6 & 0 & 0.6 \\
\hline 4 & 5 & 0.0498 & 0.2 \\
\hline 5 & 6 & 0.1990 & 0.2 \\
\hline
\end{tabular}

Test case 3: 14 BUS SYSTEM

TABLE 3.1: SYSTEM DATA

\begin{tabular}{|c|c|c|c|c|c|}
\hline $\begin{array}{c}\text { From } \\
\text { Bus }\end{array}$ & $\begin{array}{c}\text { To } \\
\text { Bus }\end{array}$ & $\begin{array}{c}\text { R } \\
\text { (p.u.) }\end{array}$ & $\begin{array}{c}\text { X } \\
\text { (p.u.) }\end{array}$ & $\begin{array}{c}\text { B/2 } \\
\text { (p.u.) }\end{array}$ & $\begin{array}{c}\text { Real Power } \\
\text { Capacity } \\
\text { (p.u.) }\end{array}$ \\
\hline 1 & 2 & 0.01938 & 0.05917 & 0.02640 & 0.6 \\
\hline 2 & 3 & 0.04699 & 0.19797 & 0.02190 & 0.7 \\
\hline 2 & 4 & 0.05811 & 0.17632 & 0.01870 & 0.8 \\
\hline 1 & 5 & 0.05403 & 0.22304 & 0.02460 & 0.5 \\
\hline 2 & 5 & 0.05695 & 0.17388 & 0.01700 & 0.4 \\
\hline 3 & 4 & 0.06701 & 0.17103 & 0.01730 & 0.3 \\
\hline 4 & 5 & 0.01335 & 0.04211 & 0.0064 & 0.2 \\
\hline 5 & 6 & 0.0 & 0.25202 & 0.0 & 0.5 \\
\hline 4 & 7 & 0.0 & 0.20912 & 0.0 & 0.4 \\
\hline 7 & 8 & 0.0 & 0.17615 & 0.0 & 0.2 \\
\hline 4 & 9 & 0.0 & 0.55618 & 0.0 & 0.2 \\
\hline 7 & 9 & 0.0 & 0.11001 & 0.0 & 0.2 \\
\hline 9 & 10 & 0.03181 & 0.08450 & 0.0 & 0.2 \\
\hline 6 & 11 & 0.09498 & 0.19890 & 0.0 & 0.3 \\
\hline 6 & 12 & 0.12291 & 0.25581 & 0.0 & 0.2 \\
\hline 6 & 13 & 0.06615 & 0.13027 & 0.0 & 0.2 \\
\hline 9 & 14 & 0.12711 & 0.27038 & 0.0 & 0.2 \\
\hline 10 & 11 & 0.8205 & 0.19207 & 0.0 & 0.2 \\
\hline 12 & 13 & 0.22092 & 0.19988 & 0.0 & 0.2 \\
\hline 13 & 14 & 0.17093 & 0.34802 & 0.0 & 0.2 \\
\hline & & & & & \\
\hline
\end{tabular}

\begin{tabular}{|c|c|c|}
\hline Transformer & Between Buses & Tap Setting \\
\hline 1 & $4-7$ & 0.978 \\
\hline 2 & $4-9$ & 0.969 \\
\hline 3 & $5-6$ & 0.932 \\
\hline
\end{tabular}




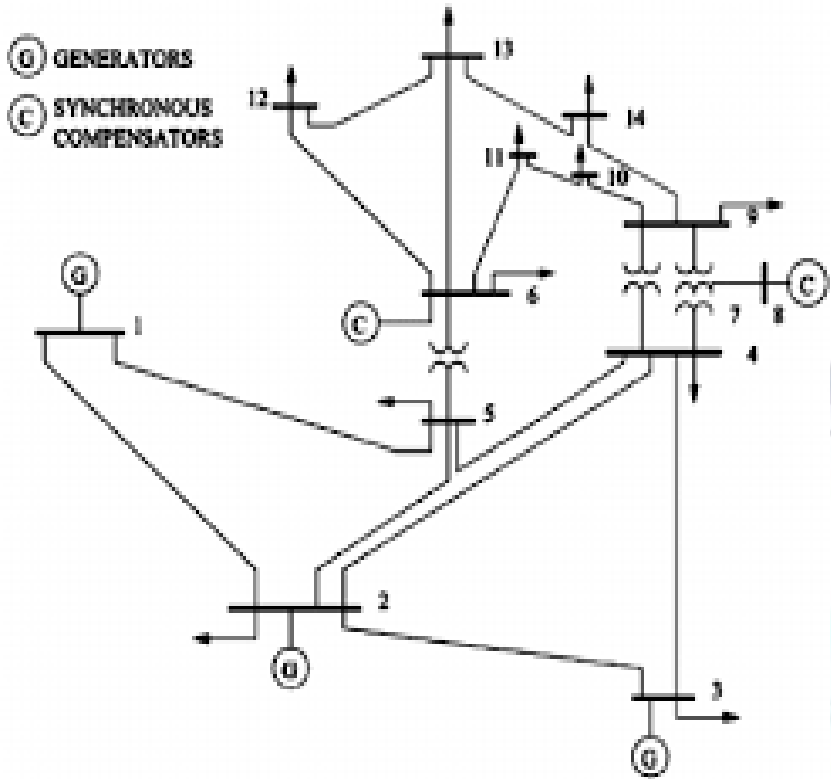

Fig. 3: Fouteen-bus Network

TABLE 3.2: RANKING OF THE LINES

\begin{tabular}{|c|c|c|c|c|}
\hline Tripped line & $\mathrm{PI}_{\mathrm{V}}$ & $\mathrm{PI}_{\mathrm{MW}}$ & $\mathrm{PI}$ & Rank \\
\hline $1-2$ & 13.8951 & 0.4008 & 14.2958 & 15 \\
\hline $2-3$ & 14.8189 & 0.2016 & 15.0204 & 10 \\
\hline $2-4$ & 14.4829 & 0.1589 & 14.6418 & 13 \\
\hline $1-5$ & 14.8244 & 0.1034 & 14.9278 & 11 \\
\hline $2-5$ & 14.8244 & 0.1034 & 14.9278 & 11 \\
\hline $3-4$ & 15.9340 & 0.0929 & 16.0269 & 2 \\
\hline $4-5$ & 14.3103 & 0.0953 & 14.4056 & 14 \\
\hline $6-11$ & 13.5478 & 0.0859 & 13.6338 & 17 \\
\hline $6-12$ & 14.1924 & 0.0849 & 14.2773 & 16 \\
\hline $6-13$ & 12.3364 & 0.1160 & 12.4524 & 20 \\
\hline $7-8$ & 12.6066 & 0.1014 & 12.7081 & 19 \\
\hline $7-9$ & 13.0923 & 0.0977 & 13.1900 & 18 \\
\hline $9-10$ & 15.0850 & 0.1025 & 15.1875 & 8 \\
\hline $9-14$ & 15.6176 & 0.1010 & 15.7186 & 4 \\
\hline $10-11$ & 15.3310 & 0.1046 & 15.4356 & 7 \\
\hline $12-13$ & 15.6541 & 0.0992 & 15.7533 & 3 \\
\hline $13-14$ & 14.9609 & 0.1023 & 15.0631 & 9 \\
\hline $4-7$ & 15.5247 & 0.1012 & 15.6259 & 5 \\
\hline $4-9$ & 15.2109 & 0.1079 & 15.3188 & 6 \\
\hline $5-6$ & 17.5626 & 0.1634 & 17.7260 & 1 \\
\hline & & & & \\
\hline
\end{tabular}

TABLE 3.3: PRE CONTINGENCY AND POST CONTINGENCY STATE BUS VOLTAGES FOR THE LINE OUTAGE (5-6)

\begin{tabular}{|c|c|c|}
\hline Bus No. & $\begin{array}{c}\text { Pre-Contingency } \\
\text { Voltage } \\
\text { (p.u.) }\end{array}$ & $\begin{array}{c}\text { Post-Contingency } \\
\text { Voltage } \\
\text { (p.u.) }\end{array}$ \\
\hline 1 & 1.0600 & 1.0600 \\
\hline 2 & 1.0450 & 1.0450 \\
\hline 3 & 1.0100 & 1.0100 \\
\hline 4 & 1.0177 & 1.0181 \\
\hline 5 & 1.0195 & 1.0272 \\
\hline 6 & 1.0700 & 1.0700 \\
\hline
\end{tabular}

\begin{tabular}{|c|l|l|}
\hline 7 & 1.0615 & 1.0656 \\
\hline 8 & 1.0900 & 1.0900 \\
\hline 9 & 1.0559 & 1.0682 \\
\hline 10 & 1.0510 & 1.0614 \\
\hline 11 & 1.0569 & 1.0623 \\
\hline 12 & 1.0552 & 1.0543 \\
\hline 13 & 1.0504 & 1.0525 \\
\hline 14 & 1.0355 & 1.0422 \\
\hline
\end{tabular}

TABLE 3.4: POST CONTINGENCY POWERFLOWS

\begin{tabular}{|c|c|c|c|}
\hline \multirow{2}{*}{$\begin{array}{l}\text { From } \\
\text { Bus }\end{array}$} & \multirow{2}{*}{$\begin{array}{c}\text { To } \\
\text { Bus }\end{array}$} & $\begin{array}{c}\text { When } 5-6 \text { line is } \\
\text { tripped }\end{array}$ & \multirow{2}{*}{$\begin{array}{c}\text { Real Power } \\
\text { Capacity } \\
\text { (p.u.) }\end{array}$} \\
\hline & & \begin{tabular}{|c|} 
Post- \\
Contingency \\
Power (p.u.)
\end{tabular} & \\
\hline 1 & 2 & 1.6147 & 0.6 \\
\hline 2 & 3 & 0.7584 & 0.7 \\
\hline 2 & 4 & 0.6166 & 0.8 \\
\hline 1 & 5 & 0.7420 & 0.5 \\
\hline 2 & 5 & 0.3771 & 0.4 \\
\hline 3 & 4 & 0.2085 & 0.3 \\
\hline 4 & 5 & 0.9963 & 0.2 \\
\hline 5 & 6 & 0.0000 & 0.5 \\
\hline 4 & 7 & 0.5613 & 0.4 \\
\hline 7 & 8 & 0.0000 & 0.2 \\
\hline 4 & 9 & 0.3191 & 0.2 \\
\hline 7 & 9 & 0.5739 & 0.2 \\
\hline 9 & 10 & 0.3349 & 0.2 \\
\hline 6 & 11 & 0.1969 & 0.3 \\
\hline 6 & 12 & 0.0455 & 0.2 \\
\hline 6 & 13 & 0.0394 & 0.2 \\
\hline 9 & 14 & 0.2733 & 0.2 \\
\hline 10 & 11 & 0.2418 & 0.2 \\
\hline 12 & 13 & -0.0159 & 0.2 \\
\hline 13 & 14 & -0.1127 & 0.2 \\
\hline
\end{tabular}

Test case 4: 30 BUS SYSTEM

TABLE 4.1: SYSTEM DATA

\begin{tabular}{|c|c|c|c|c|c|}
\hline $\begin{array}{c}\text { Fro } \\
\mathrm{m} \\
\text { Bus }\end{array}$ & $\begin{array}{c}\text { To } \\
\text { Bus }\end{array}$ & $\begin{array}{c}\mathrm{R} \\
\text { (p.u.) }\end{array}$ & $\begin{array}{c}\mathrm{X} \\
\text { (p.u.) }\end{array}$ & $\begin{array}{c}\text { B/2 } \\
\text { (p.u.) }\end{array}$ & $\begin{array}{c}\text { Real Power } \\
\text { Capacity } \\
\text { (p.u.) }\end{array}$ \\
\hline 1 & 2 & 0.0192 & 0.0575 & 0.0528 & 1.0400 \\
\hline 1 & 3 & 0.0452 & 0.1652 & 0.0408 & 1.0400 \\
\hline 2 & 4 & 0.0570 & 0.1737 & 0.0368 & 0.5200 \\
\hline 3 & 4 & 0.0132 & 0.0379 & 0.0084 & 1.0400 \\
\hline 2 & 5 & 0.0472 & 0.1983 & 0.0418 & 1.0400 \\
\hline 2 & 6 & 0.0581 & 0.1763 & 0.0374 & 0.5200 \\
\hline 4 & 6 & 0.0119 & 0.0414 & 0.0090 & 0.7200 \\
\hline 5 & 7 & 0.0460 & 0.1160 & 0.0204 & 0.5600 \\
\hline 6 & 7 & 0.0267 & 0.0820 & 0.0170 & 1.0400 \\
\hline 6 & 8 & 0.0120 & 0.0420 & 0.0090 & 0.2560 \\
\hline 6 & 9 & 0.0 & 0.2080 & 0.0 & 0.5200 \\
\hline
\end{tabular}




\begin{tabular}{|c|c|c|c|c|c|}
\hline 6 & 10 & 0.0 & 0.5560 & 0.0 & 0.2560 \\
\hline 9 & 10 & 0.0 & 0.1100 & 0.0 & 0.5200 \\
\hline 4 & 12 & 0.0 & 0.2560 & 0.0 & 0.5200 \\
\hline 12 & 14 & 0.1231 & 0.2559 & 0.0 & 0.2560 \\
\hline 12 & 15 & 0.0662 & 0.1304 & 0.0 & 0.2560 \\
\hline 12 & 16 & 0.0945 & 0.1987 & 0.0 & 0.2560 \\
\hline 14 & 15 & 0.2210 & 0.1997 & 0.0 & 0.1280 \\
\hline 16 & 17 & 0.0524 & 0.1923 & 0.0 & 0.1280 \\
\hline 15 & 18 & 0.1073 & 0.2185 & 0.0 & 0.1280 \\
\hline 18 & 19 & 0.0639 & 0.1292 & 0.0 & 0.1280 \\
\hline 19 & 20 & 0.0340 & 0.0680 & 0.0 & 0.2560 \\
\hline 10 & 20 & 0.0936 & 0.2090 & 0.0 & 0.2560 \\
\hline 10 & 17 & 0.0324 & 0.0845 & 0.0 & 0.2560 \\
\hline 10 & 21 & 0.0348 & 0.0749 & 0.0 & 0.2560 \\
\hline 10 & 22 & 0.0727 & 0.1499 & 0.0 & 0.2560 \\
\hline 21 & 22 & 0.0116 & 0.0236 & 0.0 & 0.2560 \\
\hline 15 & 23 & 0.1000 & 0.2020 & 0.0 & 0.1280 \\
\hline 22 & 24 & 0.1150 & 0.1790 & 0.0 & 0.1280 \\
\hline 23 & 24 & 0.1320 & 0.2700 & 0.0 & 0.1280 \\
\hline 24 & 25 & 0.1885 & 0.3292 & 0.0 & 0.4800 \\
\hline 25 & 27 & 0.1093 & 0.2087 & 0.0 & 0.1280 \\
\hline 28 & 27 & 0.0 & 0.3960 & 0.0 & 0.5200 \\
\hline 27 & 29 & 0.2198 & 0.4153 & 0.0 & 0.1280 \\
\hline 27 & 30 & 0.3202 & 0.6027 & 0.0 & 0.1280 \\
\hline 29 & 30 & 0.2399 & 0.4533 & 0.0 & 0.1280 \\
\hline 8 & 28 & 0.0636 & 0.2000 & 0.4028 & 0.2560 \\
\hline 6 & 28 & 0.0169 & 0.0599 & 0.0130 & 0.2560 \\
\hline 9 & 11 & 0.0 & 0.2080 & 0.0 & 0.5200 \\
\hline 12 & 13 & 0.0 & 0.1400 & 0.0 & 0.5200 \\
\hline 25 & 26 & 0.2544 & 0.3800 & 0.0 & 0.1280 \\
\hline & & & & & \\
\hline
\end{tabular}

TABLE 4.2: RANKING OF THE LINES

\begin{tabular}{|c|c|c|c|c|}
\hline $\begin{array}{c}\text { Tripped } \\
\text { line }\end{array}$ & PIv & $\mathrm{PI}_{\mathrm{MW}}$ & PI & Rank \\
\hline $1-2$ & 15.7079 & 0.1711 & 15.8791 & 33 \\
\hline $1-3$ & 15.5330 & 0.0479 & 15.5809 & 37 \\
\hline $2-4$ & 15.9123 & 0.0539 & 15.9661 & 31 \\
\hline $3-4$ & 16.4893 & 0.0606 & 16.5499 & 18 \\
\hline $2-5$ & 15.9673 & 0.0785 & 16.0458 & 30 \\
\hline $2-6$ & 15.9956 & 0.0529 & 16.0484 & 29 \\
\hline $4-6$ & 16.4688 & 0.0451 & 16.5139 & 19 \\
\hline $5-7$ & 16.2322 & 0.0418 & 16.2740 & 25 \\
\hline $6-7$ & 16.7208 & 0.0377 & 16.7585 & 16 \\
\hline $6-8$ & 16.0930 & 0.0470 & 16.1400 & 28 \\
\hline $6-9$ & 17.8893 & 0.0456 & 17.9348 & 5 \\
\hline $6-10$ & 15.1647 & 0.0433 & 15.2081 & 39 \\
\hline $9-11$ & 14.8014 & 0.0575 & 14.8589 & 41 \\
\hline $9-10$ & 14.9948 & 0.0524 & 15.0472 & 40 \\
\hline $4-12$ & 17.0075 & 0.0518 & 17.0593 & 9 \\
\hline $12-13$ & 17.7687 & 0.0690 & 17.8377 & 8 \\
\hline $12-14$ & 15.6947 & 0.0483 & 15.7430 & 35 \\
\hline $12-15$ & 16.3546 & 0.0514 & 16.4060 & 20 \\
\hline $12-16$ & 15.5754 & 0.0473 & 15.6227 & 36 \\
\hline 14-15 & 16.2498 & 0.0445 & 16.2943 & 23 \\
\hline 16-17 & 16.2244 & 0.0448 & 16.2692 & 26 \\
\hline $15-18$ & 15.9033 & 0.0448 & 15.9481 & 32 \\
\hline 18-19 & 16.1722 & 0.0445 & 16.2167 & 27 \\
\hline $19-20$ & 16.6997 & 0.0469 & 16.7466 & 17 \\
\hline $10-20$ & 16.8525 & 0.0498 & 16.9022 & 15 \\
\hline $10-17$ & 15.7424 & 0.0468 & 15.7892 & 34 \\
\hline $10-21$ & 16.9585 & 0.0516 & 17.0101 & 12 \\
\hline $10-22$ & 16.3407 & 0.0485 & 16.3892 & 21 \\
\hline $21-22$ & 16.2294 & 0.0461 & 16.2755 & 24 \\
\hline $15-23$ & 17.8131 & 0.0487 & 17.8619 & 7 \\
\hline $22-24$ & 20.7223 & 0.0495 & 20.7718 & 4 \\
\hline $23-24$ & 17.0005 & 0.0476 & 17.0481 & 11 \\
\hline $24-25$ & 24.0833 & 0.0472 & 24.1305 & 1 \\
\hline $25-26$ & 15.2487 & 0.0422 & 15.2909 & 38 \\
\hline $25-27$ & 17.8429 & 0.0458 & 17.8887 & 6 \\
\hline $27-28$ & 17.0075 & 0.0518 & 17.0593 & 9 \\
\hline $27-29$ & 22.2469 & 0.0502 & 22.2971 & 2 \\
\hline $27-30$ & 21.9454 & 0.0502 & 21.9956 & 3 \\
\hline $29-30$ & 16.9006 & 0.0422 & 16.9428 & 14 \\
\hline $8-28$ & 16.3068 & 0.0600 & 16.3668 & 22 \\
\hline $6-28$ & 16.9376 & 0.0585 & 16.9961 & 13 \\
\hline
\end{tabular}

TABLE 4.3: PRE CONTINGENCY AND POST CONTINGENCY STATE BUS VOLTAGES FOR THE LINE OUTAGE (24-25)

\begin{tabular}{|c|c|c|}
\hline Bus No. & $\begin{array}{c}\text { Pre-Contingency } \\
\text { Voltage } \\
\text { (p.u.) }\end{array}$ & $\begin{array}{c}\text { Post-Contingency } \\
\text { Voltage } \\
\text { (p.u.) }\end{array}$ \\
\hline 1 & 1.0500 & 1.0500 \\
\hline 2 & 1.0338 & 1.0338 \\
\hline 3 & 1.0290 & 1.0291 \\
\hline 4 & 1.0236 & 1.0237 \\
\hline 5 & 1.0058 & 1.0058 \\
\hline 6 & 1.0219 & 1.0216 \\
\hline
\end{tabular}




\begin{tabular}{|c|c|c|}
\hline 7 & 1.0075 & 1.0074 \\
\hline 8 & 1.0230 & 1.0230 \\
\hline 9 & 1.0418 & 1.0443 \\
\hline 10 & 1.0356 & 1.0409 \\
\hline 11 & 1.0913 & 1.0913 \\
\hline 12 & 1.0432 & 1.0457 \\
\hline 13 & 1.0883 & 1.0883 \\
\hline 14 & 1.0280 & 1.0317 \\
\hline 15 & 1.0230 & 1.0280 \\
\hline 16 & 1.0327 & 1.0364 \\
\hline 17 & 1.0293 & 1.0341 \\
\hline 18 & 1.0152 & 1.0203 \\
\hline 19 & 1.0137 & 1.0189 \\
\hline 20 & 1.0184 & 1.0236 \\
\hline 21 & 1.0212 & 1.0284 \\
\hline 22 & 1.0211 & 1.0289 \\
\hline 23 & 1.0099 & 1.0196 \\
\hline 24 & 1.0010 & 1.0169 \\
\hline 25 & 0.9797 & 0.9412 \\
\hline 26 & 0.9613 & 0.9220 \\
\hline 27 & 0.9753 & 0.9506 \\
\hline 28 & 1.0208 & 1.0177 \\
\hline 29 & 0.9544 & 0.9290 \\
\hline 30 & 0.9423 & 0.9166 \\
\hline & & \\
\hline
\end{tabular}

TABLE 4.4: POST CONTINGENCY POWERFLOWS

\begin{tabular}{|c|c|c|c|}
\hline \multirow{2}{*}{$\begin{array}{c}\text { From } \\
\text { Bus }\end{array}$} & \multirow{2}{*}{$\begin{array}{l}\text { To } \\
\text { Bus }\end{array}$} & $\begin{array}{l}\text { When } 24-25 \\
\text { line is tripped }\end{array}$ & \multirow{2}{*}{$\begin{array}{c}\text { Real Power } \\
\text { Capacity } \\
\text { (p.u.) }\end{array}$} \\
\hline & & $\begin{array}{c}\text { Post- } \\
\text { Contingency } \\
\text { Power (p.u.) }\end{array}$ & \\
\hline 1 & 2 & 0.9077 & 1.0400 \\
\hline 1 & 3 & 0.4786 & 1.0400 \\
\hline 2 & 4 & 0.2920 & 0.5200 \\
\hline 3 & 4 & 0.4452 & 1.0400 \\
\hline 2 & 5 & 0.5814 & 1.0400 \\
\hline 2 & 6 & 0.3785 & 0.5200 \\
\hline 4 & 6 & 0.3877 & 0.7200 \\
\hline 5 & 7 & 0.1300 & 0.5600 \\
\hline 6 & 7 & 0.3623 & 1.0400 \\
\hline 6 & 8 & 0.0093 & 0.2560 \\
\hline 6 & 9 & 0.1522 & 0.5200 \\
\hline 6 & 10 & 0.1204 & 0.2560 \\
\hline 9 & 10 & 0.3292 & 0.5200 \\
\hline 4 & 12 & 0.2698 & 0.5200 \\
\hline 12 & 14 & 0.0776 & 0.2560 \\
\hline 12 & 15 & 0.1777 & 0.2560 \\
\hline 12 & 16 & 0.0682 & 0.2560 \\
\hline 14 & 15 & 0.0148 & 0.1280 \\
\hline 16 & 17 & 0.0328 & 0.1280 \\
\hline 15 & 18 & 0.0568 & 0.1280 \\
\hline 18 & 19 & 0.0244 & 0.1280 \\
\hline 19 & 20 & 0.0706 & 0.2560 \\
\hline 10 & 20 & 0.0937 & 0.2560 \\
\hline 10 & 17 & 0.0575 & 0.2560 \\
\hline
\end{tabular}

\begin{tabular}{|c|c|c|c|}
\hline 10 & 21 & 0.1645 & 0.2560 \\
\hline 10 & 22 & 0.0805 & 0.2560 \\
\hline 21 & 22 & 0.0117 & 0.2560 \\
\hline 15 & 23 & 0.0517 & 0.1280 \\
\hline 22 & 24 & 0.0682 & 0.1280 \\
\hline 23 & 24 & 0.0194 & 0.1280 \\
\hline 24 & 25 & 0 & 0.4800 \\
\hline 25 & 27 & 0.0355 & 0.1280 \\
\hline 28 & 27 & 0.1620 & 0.5200 \\
\hline 27 & 29 & 0.0621 & 0.1280 \\
\hline 27 & 30 & 0.0712 & 0.1280 \\
\hline 29 & 30 & 0.0371 & 0.1280 \\
\hline 8 & 28 & 0.0407 & 0.2560 \\
\hline 6 & 28 & 0.1288 & 0.2560 \\
\hline 9 & 11 & 0.1793 & 0.5200 \\
\hline 12 & 13 & 0.1691 & 0.5200 \\
\hline 25 & 26 & 0.0355 & 0.1280 \\
\hline
\end{tabular}

\section{CONCLUSION}

To give the contingency ranking, performance index has been used as a measuring tool. Newton Raphson power flow method is found most appropriate method in the approach of contingency selection, as it played a vital role in rejecting the large number of contingency cases and concentrated on the most severe contingency case. From the results, it is seen that the calculation of performance indices gives a good measure of the cases, which has the highest potential to form the system

parameters to go beyond their limits, calculation of PI are also obtained for the sake of increase in the accuracy of the sorting and ranking technique of the contingency analysis process.

\section{FUTURE SCOPE}

From the above one can judge the state of power system if any equipment gets outage from the system. So by forecasting the effect of any outage, the operator come to know the prior action what should be done for the particular outage. Especially at this condition "FACT" devices will help to control the power flow and to maintain good voltage profile at each and every bus. TCSC will be used to control the power flow through the lines which is getting overloaded and ST ATCOM, SVC will be used to maintain good voltage profile by giving

reactive power or by taking reactive power from the bus. So we can extend this work by placing "FACT" devices in our system. 


\section{REFERENCES}

[1] L.H. Fink and K. Carlsen, "Operating under stress and strain," IEEE Spectrum; (United States\}. vol. 15, 1978.

[2] K.S. Swarup and G. Sudhakar, "Neural network approach to contingency screening and ranking in power systems," Neurocomputing, vol. 70, pp. 105-1 18, 2006.

[3] G. Ejebe and B. Wollenberg, "Automatic contingency selection," Power Apparatus and Systems. IEEE Transactions on, pp. 97-109, 1979.

[4] B. Stott, O. Alsac, and A. J. Monticelli, "Security analysis and optimization," Proceedings of the IEEE, vol. 75, pp. 1623-1644, 1987.

[5] A.J. Wood and B.F. Wollenberg, Power generation. operation. And control: John Wiley \& Sons, 2012.

[6] J. Zaborszky, K.W. Whang, and K. Prasad, "Fast contingency evaluation using concentric relaxation," Power Apparatus and Systems. IEEE Transactions on, pp. 28-36, 1980.

[7] C.Y. Lee and N. Chen, "Distribution factors of reactive power flow in transmission line and transformer outage studies," Power Systems. IEEE Transactions on, vol. 7, pp. 194-200, 1992.

[8] F. Albuyeh, A. Bose, and B. Heath, "Reactive power considerations in automatic contingency selection," Power Apparatus and Systems. IEEE Transactions on, pp. 107-112, 1982.

[9] S. Naik, M. Khedkar, and S. Bhat, "Effect of line contingency on static voltage stability and maximum loadability in large multi bus power system," International Journal of Electrical Power \& Energy Systems, vol. 67, pp. 448-452, 2015. 